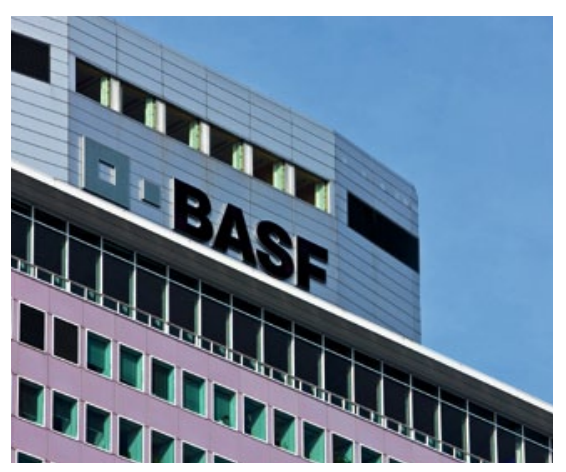

\section{TDI-Geschäft abgeschlossen}

BASF konnte am 11. März 2013 die Akquisition von Teilen des TDI-Geschäfts (Toluoldiisocyanat) von Ciech erfolgreich abschließen. Die TDI-Produktionsanlage der Ciech-Tochtergesellschaft Zachem in Polen ist von der Transaktion nicht betroffen.

„Mit dieser Akquisition bauen wir unsere führende Position im IsocyanateMarkt weiter aus“, sagte Kenneth Lane, Leiter der Geschäftseinheit Globales Strategisches Marketing PolyurethanBasisprodukte Europa. „Oberste Priorität hat nun die reibungslose Integration für unsere Kunden und Mitarbeiter." BASF betreibt TDI-Anlagen in Geismar/Louisiana, Yeosu/Südkorea, Caojing/China, Schwarzheide/ Deutschland und ab 2014 in Ludwigshafen/Deutschland.

\title{
M\&A-Geschäft in der Chemieindustrie zieht wieder an
}

Einer aktuellen Umfrage zufolge, die die Unternehmensberatung A.T. Kearney im vierten Quartal in der internationalen Chemieindustrie durchgeführt hat, wird das M\&A-Geschäft in diesem Jahr wieder Fahrt aufnehmen. 2012 war für die weltweite Chemieindustrie ein vergleichsweise schwaches Jahr für Übernahmen und Fusionen.

E $\begin{aligned} & \text { ast zwei Drittel der Befragten erach- } \\ & \text { ten die günstigen Finanzierungs- }\end{aligned}$ konditionen als Haupttreiber hinter dieser Entwicklung. Dies könnte bedeuten, dass strategische Investoren ihre wiedererlangte finanzielle Schlagkraft nutzen werden, um regional zu expandieren.

\section{Unterschiedlich hohe Aktivitätslevel}

Für die verschiedenen Regionen werden unterschiedlich hohe Aktivitätslevel erwartet. Über 90 Prozent der Befragten erwarten eine weitere Konsolidierung der stark fragmentierten asiatischen Märkte, was die M\&A-Aktivität in China, Indien und dem Rest Asiens anfachen dürfte. Im Gegensatz dazu ist der Ausblick für Europa gemischt. 70 Prozent der Befragten erachten die wirtschaftliche Unsicherheit und die Wirtschaftskrise als hinderlich. Für 57 Prozent der Befragten stellt der Zugang zu kostengünstigen Rohstoffen in den USA, Kanada und Me- xiko einen Treiber für M\&A-Aktivitäten im Jahr 2013 dar.

\section{Neue Formen der \\ Zusammenarbeit}

Weiterhin ist der Studie zu entnehmen, dass mehr als die Hälfte der Befragten eine Zunahme der Kooperationsformen jenseits von M\&A erwartet - etwa in Form von Joint Ventures, langfristigen Lieferabkommen und strategischen Allianzen.

Die Analysten sehen derzeit drei wesentliche Treiber für neue Partnerschaftsmodelle: Zugang zu Rohstoffen, Märkten und Technologien. Neue Partnerschaften werden sich vor allem im Rahmen von Industrie-Neuansiedlungen in neuen Regionen entwickeln, bei denen alternative Rohstoffe zum Einsatz kommen und sich der Zugang zu herkömmlichen Rohstoffen mit technologischem Know-how bzw. dem Zugang zu Märkten verbinden lässt.

Weitere Infos: www.atkearney.de

\section{Seminar: Verklebung von Glas, Kunststoffen und Metall}

Unter dem Titel „Verklebung von Glas, Kunststoffen / Composite und Metall Know-how, Erfahrung und Praxis aus dem Automobilbau und angrenzenden Industrien“ stellen Bodo Möller Chemie und der Partner Dow Automotive Systems am 6. Juni 2013 in Esslingen die wachsende Bedeutung spezialisierter Klebstoffe als Innovationsträger im Automobilbau und zahlreichen weiteren Branchen vor.
Dieses Seminar soll die Innovationskraft und die erhöhte Konstruktionsfreiheit moderner Klebstoffe vermitteln - insbesondere in Bereichen wie dem Automobil-, Transport- und Nutzfahrzeugbau. Durch spezialisierte Klebund Dichtstoffe sowie Vorbehandlungssysteme und Mischer lassen sich Glas, Metalle, Kunststoffe und auch Composites einfach und absolut zuverlässig miteinander verbinden. Die Nut- zung neuartiger Werkstoffe - etwa bei Leichtbauweisen - wird durch neue Entwicklungen der Klebstoffindustrie seit Jahren gefördert.

Das Seminar richtet sich sowohl an Interessenten, die Klebstoffe als moderne Fügetechnologie neu kennenlernen wollen, aber auch solche, die bereits vorhandene Kenntnisse vertiefen wollen.

Weitere Infos: www.bm-chemie.de 